\title{
Nanowire single-photon detectors made of atomic layer-deposited niobium nitride
}

\author{
E. Knehr ${ }^{1,2}$, A. Kuzmin ${ }^{2}$, M. Ziegler ${ }^{1}$, S. Doerner ${ }^{2}$, \\ K. Ilin $^{2}$, M. Siegel ${ }^{2}$, R. Stolz ${ }^{1}$ and H. Schmidt ${ }^{1,3}$ \\ ${ }^{1}$ Department of Quantum Detection, Leibniz Institute of Photonic Technology \\ (Leibniz IPHT), Jena, Germany \\ ${ }^{2}$ Institute of Micro- and Nanoelectronic Systems, Karlsruhe Institute of \\ Technology (KIT), Karlsruhe, Germany \\ ${ }^{3}$ Institute for Solid State Physics, University of Jena, Jena, Germany \\ E-mail: emanuel.knehr@leibniz-ipht.de
}

\begin{abstract}
We demonstrate and characterize first superconducting nanowire single-photon detectors (SNSPDs) made from atomic layer- deposited (ALD) NbN layers. To assess the suitability of these films as a detector material, transport properties of bare films and bridges of different dimensions and thicknesses are investigated. Similar ratios of the measured critical current to the depairing current are obtained for micro-bridges made from ALD and sputtered NbN films. Furthermore, we characterized the single-photon response for 5 and $10 \mathrm{~nm}$ thick nanowire detectors. A $100 \mathrm{~nm}$-wide straight nanowire with a length of $5 \mu \mathrm{m}$ exhibits saturated count-rate dependencies on bias current and a cut-off wavelength in the near-infrared range. The ALD technique could open up the possibility to fabricate NbN-based detectors on the wafer scale and to conformally cover also non-planar surfaces for novel device concepts.
\end{abstract}

Keywords: atomic layer deposition, superconducting detectors, single-photon detector, superconducting nanowire, niobium nitride 


\section{Introduction}

Being developed since their introduction in 2001 1, superconducting nanowire single-photon detectors (SNSPDs) are very promising devices for applications in fields such as quantum cryptography, spectroscopy, and deep space communication 2 4 .

Key properties of SNSPDs include the spectral detection efficiency and the timing resolution limited by the timing jitter. By means of material research and optimization of the detector geometry and readout electronics, detection efficiencies of over $90 \%$ at $1550 \mathrm{~nm}$ and a timing resolution as low as $3 \mathrm{ps}$ have been shown recently [5, 6]. This sets SNSPDs apart from competing single-photon detectors (see table 1.1 in [7]).

For the use in SNSPDs, materials with a low superconducting energy gap, a small diffusion coefficient of the quasi-particles, and a critical current of nanowires close to the depairing current are advantageous [8, 9. Especially the latter is necessary for a longer cut-off wavelength, but is often limited by inhomogeneities and constrictions in the active area of the detector [10,11. Also, arrays of single-photon detectors require large-area ultra-thin films with a high uniformity with respect to the superconducting order parameter.

Besides recently emerging materials such as WSi [12] and MoSi [13, $\mathrm{NbN}$ is still one of the most used materials because of its relatively high critical current densities and the established deposition technology.

So far, NbN-based SNSPDs are predominantly fabricated by reactive-magnetron sputtering of niobium in a nitrogen atmosphere. However, this technology cannot ensure high uniformity and homogeneity of the NbN films and is hard to apply for larger wafer sizes. Atomic layer deposition (ALD), on the other hand, could alleviate these problems by its chemical nature, depositing superconducting thin films monolayer by monolayer while making lower demands on the vacuum system and the temperature homogeneity of the substrate. Furthermore, other features of this deposition technique like the conformal coverage of pre-patterned substrates could lead to new applications.

Previously, ALD TiN films were reported for the application in SNSPDs [14. In our earlier work, the deposition of superconducting $\mathrm{NbN}$ thin films using metal-organic plasma-enhanced atomic layer deposition (PEALD) has been shown [15-17]. Here, we investigate the suitability of these films for detector applications and demonstrate first SNSPDs and their properties.

\section{Fabrication technology}

We successfully deposited superconducting $\mathrm{NbN}$ in an Oxford Plasma Technology OpAL ALD system using (tert-butylimido)-tris(diethylamino)-niobium (TBTDEN) and hydrogen plasma as precursors. The substrates are alternately exposed to these precursors to deposit monolayers of TBTDEN on the surface and reduce the monolayers to form $\mathrm{NbN}$, respectively. In order to prevent gaseous phase reactions and limit the process to surface reactions, purge steps with argon as an inert gas are introduced between the precursor steps. By using a nitrogen glove box for sample handling, oxygen contaminations in the reactor could be minimized [16. The best film quality was achieved at a substrate temperature of $350^{\circ} \mathrm{C}$, a plasma power of $300 \mathrm{~W}$, and a hydrogen flow rate of $2.5 \mathrm{sccm}$. The resulting film growth per cycle was $0.46 \AA$. More details on the deposition process can be found in [15,16. The NbN films were deposited on $10 \mathrm{~mm} \times 10 \mathrm{~mm} \mathrm{R}$ plane, double-side polished sapphire substrates and subsequently spin-coated with $90 \mathrm{~nm}$ of poly-methylmethacrylate (PMMA, $950 \mathrm{k}$ ).

Using electron-beam lithography, the resist was patterned in several steps. First, the nanowires were patterned in a negative-PMMA process similar to the process shown in [11. After removing the unexposed areas with acetone, a new layer of PMMA was spun on and exposed in a positive process to pattern the readout lines, in which the detector is embedded. In a single step, the samples were etched via Ar-ion milling. After etching, the resist was again removed by acetone and 2-propanol.

The magnetron sputtering process, used to prepare $\mathrm{NbN}$ bridges for comparison (see section 3.2), is described in [18.

\section{Transport measurements}

The bare films and test bridges were characterized by standard four-point measurements in a dipstick cooled down to $4.2 \mathrm{~K}$ using liquid helium (lHe). For magnetic field measurements, a pulse tube cryocooler equipped with a superconducting magnet was used. The coils create a magnetic field up to $5 \mathrm{~T}$ perpendicular to the substrate surface of the samples.

\subsection{NbN films}

The basic electrical characteristics of bare ALD-NbN films were investigated on samples with a thickness between 3 and $21 \mathrm{~nm}( \pm 0.2 \mathrm{~nm})$.

In figure 1(a), the sheet resistance $R_{\mathrm{s}}$ in the normal state near the superconducting transition is plotted as a function of the thickness $d$. In addition, the residual resistivity $\rho_{0}=R_{\mathrm{s}} d$ is shown. The lowest 

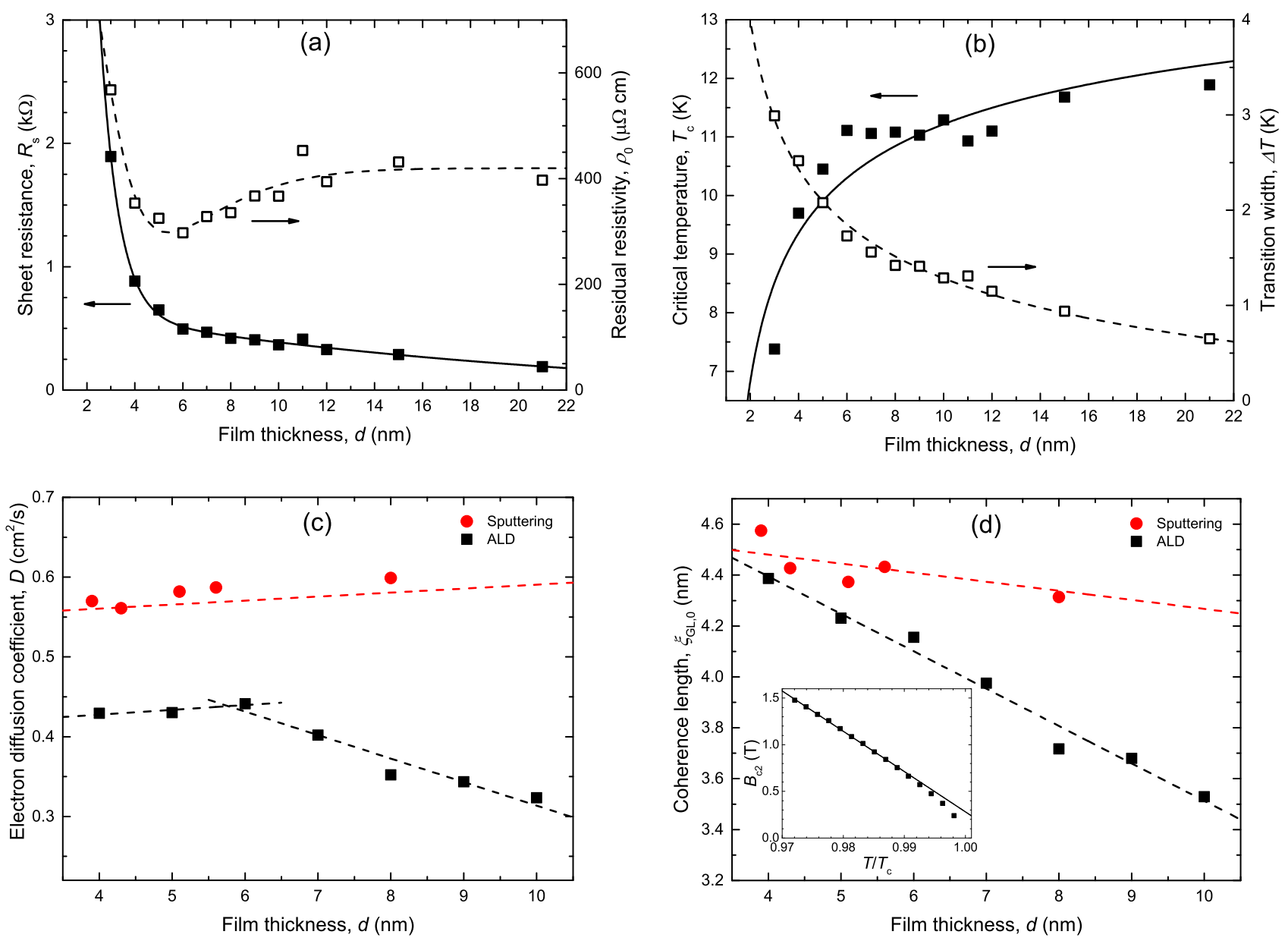

Figure 1: Electrical properties of ALD-NbN films with varying thickness; the sheet resistance $R_{\mathrm{S}}$ and residual resistivity $\rho_{0}$ in normal state (a), the critical temperature $T_{\mathrm{c}}$ and the transition width $\Delta T$ (b), the electron diffusion coefficient $D(\mathrm{c})$, and the Ginzburg-Landau coherence length at zero temperature $\xi_{\mathrm{GL}}(0)(\mathrm{d})$. The solid and dashed lines are to guide the eye. The comparison values for sputtered films are taken from [19. (Inset) Temperature dependence of the upper critical magnetic field. The line represents a linear fit excluding the data points above $0.99 \mathrm{~T} / T_{\mathrm{c}}$.

value for $\rho_{0}$ of about $300 \mu \Omega \mathrm{cm}$ corresponds to a film thickness of $6 \mathrm{~nm}$. For film thicknesses larger than $10 \mathrm{~nm}, \rho_{0}$ is almost constant, while it rapidly increases for $d<4 \mathrm{~nm}$.

In figure $1(\mathrm{~b})$, both the critical temperature $T_{\mathrm{c}}$ (taken at $0.01 R_{\mathrm{S}}$ ) and the width of the transition $\Delta T$ (temperature difference of 0.9 and $0.1 R_{\mathrm{s}}$ ) between the superconducting and the normal state are plotted over thickness $d$. Similar to the trend of $R_{\mathrm{s}}$, the critical temperature $T_{\mathrm{c}}$ reaches a plateau above $d=6 \mathrm{~nm}$. A value of $T_{\mathrm{c}}=11.3 \mathrm{~K}$ for a $6 \mathrm{~nm}$ thick film has been obtained, while the critical temperature decreases rapidly for smaller film thicknesses, reaching $7.4 \mathrm{~K}$ at $3 \mathrm{~nm}$.

The magnetic penetration depth $\lambda$ for 5 and $10 \mathrm{~nm}$-thick films, estimated from $\mu_{0} \lambda^{2}=$ $\hbar \rho_{0} / \pi \beta_{0} k_{\mathrm{B}} T_{\mathrm{c}}\left(\beta_{0}=2.05\right.$ for NbN [20]), amounts to about $550 \mathrm{~nm}$.

To define the electron diffusion coefficient $D$ and the coherence length $\xi_{\mathrm{GL}}$, the upper critical magnetic field $B_{\mathrm{c} 2}$ has been measured as a function of the temperature (see inset in figure 1(d)). From the slope of the linear part of $B_{\mathrm{c} 2}(T)$, the coefficient $D$ was then calculated by

$D=-\frac{4 k_{\mathrm{B}}}{\pi e}\left(\frac{\mathrm{d} B_{\mathrm{c} 2}}{\mathrm{~d} T}\right)^{-1}$

with the Boltzmann constant $k_{\mathrm{B}}$ and the elementary charge $e$ (see equation (9.81) in [21]). From the linear part of $B_{\mathrm{c} 2}(T)$, the value $B_{\mathrm{c} 2}(0)$ was calculated by

$$
B_{\mathrm{c} 2}(0)=\left.0.69 T_{\mathrm{c}} \frac{\mathrm{d} B_{\mathrm{c} 2}}{\mathrm{~d} T}\right|_{T \lesssim T_{\mathrm{c}}}
$$




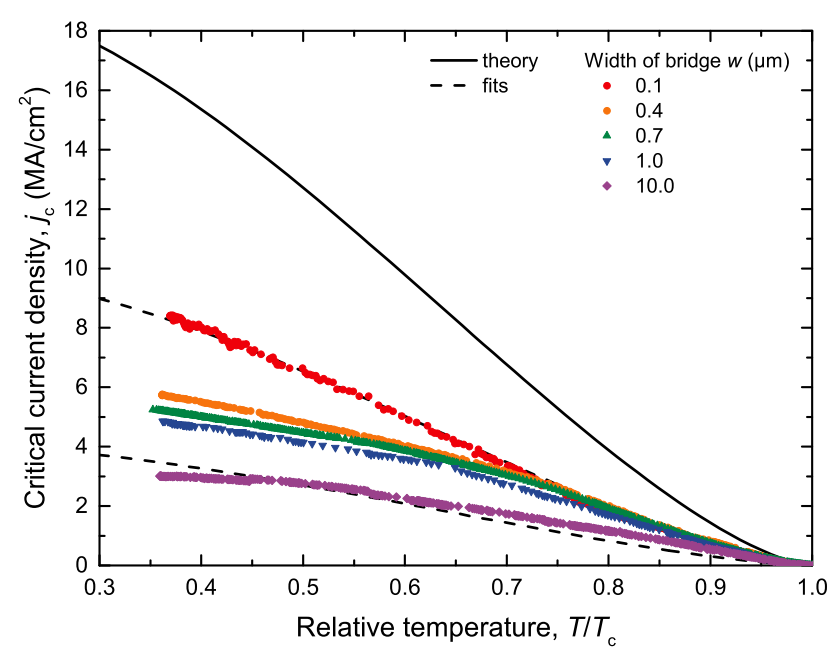

Figure 2: Dependence of the critical current density $j_{\mathrm{c}}$ on temperature $T$ normalized to the critical temperature $T_{\mathrm{c}}$ for different bridge widths and $d_{\mathrm{NbN}}=$ $10 \mathrm{~nm}$. The black line represents the temperature dependence of $j_{\text {dep }}$ according to equation (4) and (5), while the dashed lines are fits to the data of the smallest and widest bridge with $j_{\mathrm{dep}, \mathrm{GL}}(0)$ as fitting parameter.

(see equation (9.32) in [21, 22]). The GinzburgLandau coherence length at $0 \mathrm{~K}$ was estimated by

$\xi_{\mathrm{GL}}(0)=\sqrt{\frac{\Phi_{0}}{2 \pi B_{\mathrm{c} 2}(0)}}$

with the magnetic flux quantum $\Phi_{0}=h / 2 e$ (see equation (9.25) in 21]).

Compared to reported values for sputtered films, the residual resistivity of ALD films is about two times higher, while the diffusion coefficient is 1.3-2.0 times lower for the same thickness [19. Consequently, this should be attended by a similar single-spin density of states at the Fermi-level $N(0)=\left(2 e^{2} \rho_{0} D\right)^{-1}$. As a result, the heat capacity of the electronic system $C_{\mathrm{e}}=\left(2 \pi^{2} / 3\right) k_{\mathrm{B}}^{2} N(0) T$ and therefore the ratio $C_{\mathrm{e}} / C_{\mathrm{ph}}$ (with the phonon heat capacity $C_{\mathrm{ph}}$ ) is similar to sputtered films 23]. A larger $C_{\mathrm{e}} / C_{\mathrm{ph}}$ should facilitate the detection of photons because of the larger fraction of the energy which is transferred to the electronic system.

The diffusion coefficient $D$ is another important parameter when evaluating the influence of an absorbed photon on the superconducting order parameter. A small value for $D$ results in short thermalization times $\tau_{\text {th }}$ in a relatively small hot-spot volume, which improves the detection efficiency [23]. At $10 \mathrm{~nm}$ thickness, the calculated $D$ for ALD films is almost half the reference value for sputtered films (see figure 1(c)) [19, which confines the absorbed energy in a smaller vol-

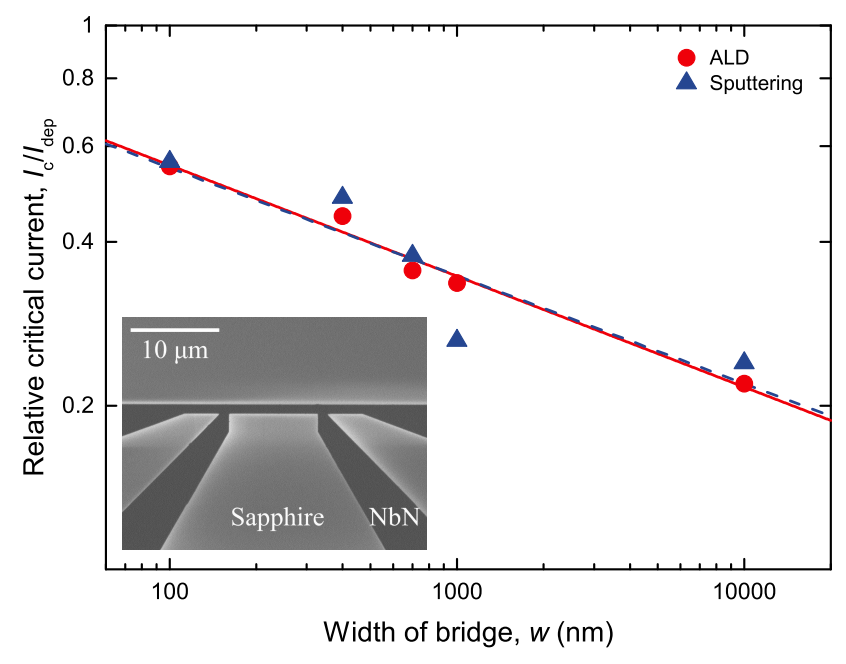

Figure 3: Dependence of the relative critical current (ratio of measured critical current $I_{\mathrm{c}}$ to calculated theoretical depairing current $I_{\mathrm{dep}}$ ) on bridge width for different deposition technologies. The film thickness was constant at $10 \mathrm{~nm}$. The solid and dashed lines are linear fits to the respective data. (Inset) Exemplary SEM image of the bridge.

ume and, subsequently, leads to a stronger suppression of the order parameter in this hotspot. The diffusion coefficient for $\mathrm{NbN}$ is expected to increase with thicker films, as the electron mean free path can be assumed to increase. Above $6 \mathrm{~nm}$, the measured $D$ differs from this expected dependence. One reason could be oxygen incorporation into the films as a result of the long ALD deposition times together with oxygen permeation into the deposition chamber. This results in growing defects (niobium oxide) in the deposited film and could lead to a decrease of the diffusion coefficient [16.

In figure 1(d), a decrease of $\xi_{\mathrm{GL}}$ with thicker films can be observed. This is expected due to the increase of $T_{\mathrm{c}}$ and the relation $\xi_{\mathrm{GL}}(0) \propto \sqrt{\xi_{0} l}$ where $\xi_{0} \propto T_{\mathrm{c}}^{-1}$ ( $\xi_{0}$ being the BCS coherence length and $l$ the mean free path of electrons, see equation (9.26) and (9.28) in [21]). The calculated value $\xi_{\mathrm{GL}}(0)=4.23 \mathrm{~nm}$ for $d=$ $5 \mathrm{~nm}$ of ALD-NbN coincides well with a reference value $\left(\xi_{\mathrm{GL}}(0) \approx 4.37 \mathrm{~nm}\right.$ for $\left.d=5.1 \mathrm{~nm}\right)$ of sputtered $\mathrm{NbN}$ films of the same thickness on sapphire substrates [19].

\section{2. $N b N$ bridges}

$\mathrm{NbN}$ bridges have been patterned to investigate the critical current densities $j_{\mathrm{c}}$. The bridges have a width $w$ between 0.1 and $10.0 \mu \mathrm{m}$ and a length $l=10 w$ (see geometry in the inset of figure 3). A reduction of the measurable critical current $I_{\mathrm{c}}$ due to a nonuniform current distribution across the bridge could be neglected, since $w \ll \Lambda$ (with the Pearl length 
$\Lambda=\left(2 \lambda^{2}\right) / d$ for thin films with $\left.d \ll \lambda\right)[24,25$.

In figure 2 , the dependence of $j_{\mathrm{c}}$ on the normalized temperature $T / T_{\mathrm{c}}$ is shown. The experimental values have been compared to the temperature dependence of the depairing critical current density $j_{\text {dep }}(T)$ according to the derivation of Kupriyanov and Lukichev [26]

$j_{\mathrm{dep}}(T)=j_{\mathrm{dep}, \mathrm{GL}}(0)\left(1-\frac{T}{T_{\mathrm{c}}}\right)^{\frac{3}{2}} \mathrm{KL}(T)$,

with the formal Ginzburg-Landau zero-temperature depairing current

$j_{\mathrm{dep}, \mathrm{GL}}(0) \approx 1.454 \beta_{0}^{2} \frac{\left(k_{\mathrm{B}} T_{\mathrm{c}}\right)^{\frac{3}{2}}}{e \rho_{0} \sqrt{D \hbar}}$

and the correction factor $\mathrm{KL}(T)$ for the dirty limit (see curve 7 , figure 1 in [26]).

In figure 3 , the ratio $I_{\mathrm{c}} / I_{\mathrm{dep}}$ is plotted for both ALD and sputtered NbN bridges. Samples of both types of deposition were patterned in parallel.

The temperature dependencies of $j_{\mathrm{c}}$ for different widths coincide near $T_{\mathrm{c}}$ as expected from the GinzburgLandau theory (figure 2). For smaller ratios $T / T_{\mathrm{c}}$, the penetration and dissipative movement of vortices across the bridge suppress the measurable value $j_{\mathrm{c}}$ for larger bridge widths.

In addition to the theoretical $j_{\text {dep }}(T)$ calculated by equation (4) and (5) (solid line, figure 2), a fit to the experimental data was also plotted (dashed lines). For these curves, we used $j_{\mathrm{dep}, \mathrm{GL}}(0)$ as a fitting parameter. As it is seen, only the data for $w=0.1 \mu \mathrm{m}$ can be well fitted by equation (4), which could indicate that the ratio $j_{\mathrm{c}} / j_{\text {dep }}$ is only reduced by constrictions. In this case, the fit estimates the relative cross-section of the superconducting core.

Although the absolute values of $j_{\mathrm{c}}$ for the ALD$\mathrm{NbN}$ bridges were significantly lower than the ones for sputtered $\mathrm{NbN}$ (9 compared to $23 \mathrm{MA} / \mathrm{cm}^{2}$ for $w=0.1 \mu \mathrm{m})$, due to a higher $\rho_{0}$ and a lower $T_{\mathrm{c}}$, the calculated ratios of $I_{\mathrm{c}}$ to $I_{\mathrm{dep}}$ are comparable, reaching almost 0.6 at the minimal width of $0.1 \mu \mathrm{m}$ (figure 3 ). According to 66,23, a higher ratio $I_{\mathrm{c}} / I_{\mathrm{dep}}$ should lead to higher internal detection efficiencies for lower-energy photons and to a smaller intrinsic timing jitter.

The obtained ratios are still below one, which is probably due to both material-inherent nonuniformities and fabrication imperfections of the nanowire geometry. Moreover, for the calculations we use the nominal width, determined by SEM, without taking the edge roughness into account. Also, damaged normal-conducting bands around the superconducting core were neglected. These are expected due to film oxidation, the formation of an intermediate layer between the substrate and the film and damaged edges during etching, as described in [27.

\section{Nanowire detectors}

We fabricated straight nanowires with a width $w=$ $100 \mathrm{~nm}$ and a length $l=5 \mu \mathrm{m}$ for the investigation of detector properties. Detectors of both $5 \mathrm{~nm}$ and $10 \mathrm{~nm}$ film thickness have been characterized. To minimize current-crowding [28, the nanowire is connected by short tapers at both ends. Since the low kinetic inductance of these short nanowires would lead to latching, a $1 \mu \mathrm{m}$-wide, $1 \mathrm{~mm}$-long inductor in series to the nanowire was patterned, adding a kinetic inductance of about $L_{\mathrm{kin}} \approx 46 \mathrm{nH}$ for $d=10 \mathrm{~nm}$ $\left(L_{\mathrm{kin}}=\mu_{0} \lambda^{2} l / w d\right.$, see e.g. equation (9.45) in [21]). The detectors have critical temperatures in the range of 9 to $11 \mathrm{~K}$ and critical currents of 15 and $43 \mu \mathrm{A}$ for $d=5 \mathrm{~nm}$ and $10 \mathrm{~nm}$, respectively.

\subsection{Experimental setup}

In order to characterize the detectors optically, the samples were mounted in a dipstick cryostat equipped with an optical fiber, RF, and DC lines. The dipstick was cooled down in $1 \mathrm{He}$ to $4.2 \mathrm{~K}$. For the detector's operation, a low-noise voltage source with RC-filters connected to a room-temperature bias-tee was used. The samples were illuminated from the top by different light sources. For measurements dependent on the bias current and the optical power, the output of an LED at $385 \mathrm{~nm}$ and a laser diode at $658 \mathrm{~nm}$ was coupled on to the detector via a multimode fiber, which is specified from 400 to $2400 \mathrm{~nm}$. For spectral measurements, a halogen lamp and a monochromator for the wavelength range between 400 and $2000 \mathrm{~nm}$ were used. In order to ensure a uniform illumination of the detectors, the samples were kept at a distance of $4-5 \mathrm{~mm}$ from the fiber tip resulting in an illuminated area with a radius of about $1 \mathrm{~mm}$.

All of the light sources were calibrated using a power meter equipped with $\mathrm{Si}$ - and InGaAs-based photodiodes for the wavelength range 350 to $1100 \mathrm{~nm}$ and 800 to $1700 \mathrm{~nm}$ (Thorlabs S150C and S154C), respectively. The calibration was done at the detector plane at the bottom of the dipstick in order to take into account any wavelength-dependent loss contribution by the vacuum fiber feedtrough, the fiber itself and the output of the stripped fiber.

The count rate was measured by a pulse counter (up to $300 \mathrm{MHz}$ count rate) with a two-stage roomtemperature preamplifier connected to the RF output of the bias-tee. The resulting pulse height was in the range of 200 to $900 \mathrm{mV}$, depending on the critical current of the sample. The pulse and jitter of the detector were monitored using a real-time oscilloscope of type Agilent Technologies DSA-X 93204A.

The output optical power from the monochromator was strongly dependent on the wavelength. In or- 

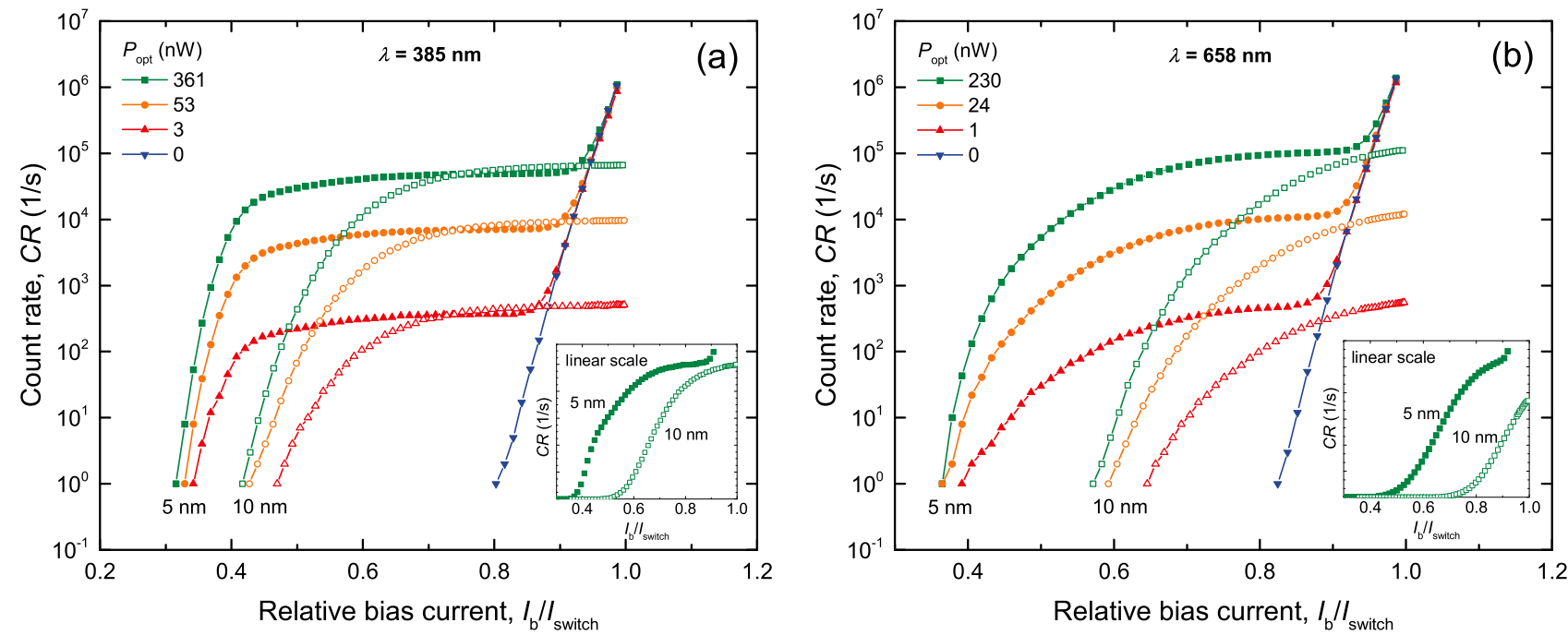

Figure 4: Dependence of the count rate $C R$ on the relative bias current $I_{\mathrm{b}} / I_{\text {switch }}$ for illumination with photons at a wavelength of $385 \mathrm{~nm}$ (a) and $658 \mathrm{~nm}$ (b) and different optical powers. (Insets) Plots on the linear scale for the highest power level.

der to keep the detector in the same detection regime during spectral measurements, the detection count rate was kept at values below $10^{4} \mathrm{~s}^{-1}$ by manually adjusting the input slit width of the monochromator accordingly. The measured data was then stitched by means of overlapping data points.

\subsection{Results}

The bias-dependent count rates for different film thicknesses, wavelengths, and optical input powers are shown in figure 4 . For $\lambda=385 \mathrm{~nm}$ (figure 4(a)), all measured curves exhibit saturating count rates, which is attributed to near-unity internal detection efficiency for high bias currents [29]. For lower bias currents, the count rate decreases exponentially. In this, so called probabilistic or fluctuation-assisted detection regime, a detection event can only occur with the help of fluctuations. As indicated by the prolonged plateau in $C R\left(I_{\mathrm{b}}\right)$, the thinner sample shows a deterministic regime over a wider bias range. At $\lambda=658 \mathrm{~nm}$ (figure $4(\mathrm{~b}))$, the plateaus are less pronounced $(5 \mathrm{~nm})$ or not existing anymore $(10 \mathrm{~nm})$. To better illustrate this, insets with curves on the linear scale are included in figure 4.

For the same optical input power, the level of saturation is largely independent of the sample thickness, further emphasizing the near-unity internal efficiency.

By blocking the feed-through of the fiber, the dark-count rate $(D C R)$ was obtained. We measured an $D C R$ exponentially growing with the bias current for $d=5 \mathrm{~nm}$ and $I_{\mathrm{b}}>0.8 I_{\text {switch }}$, whereas the $10 \mathrm{~nm}$ sample did not exhibit an increasing $D C R$ above a noise level $\leq 3 \mathrm{~s}^{-1}$ up to $I_{\text {switch }}$.

As illustrated in figure 5 , the count rate increases with the incoming photon rate $P R$ as $C R \propto P R^{n}$ with an exponent $n$ of about $1.005 \pm 0.005$. This applies to all measured $I_{\mathrm{b}} / I_{\text {switch }}$-ratios corresponding to different bias regimes. The linear dependence of $C R \propto P R$ over many orders of magnitude is a strong indication of single-photon detection [1, 30. A saturation of the count rate $C R_{\max }$ could not be reached because of the weak optical coupling and the subsequent heating of the sample, suppressing $I_{\text {switch }}$ and therefore limiting $I_{\mathrm{b}}$. For $I_{\mathrm{b}}>0.81 I_{\text {switch }}$ and low photon rates, the count rate converges to $D C R$. The divergence of the data points with respect to the linear progression at lower $P R$ is due to the calibration precision of the power meter (about $100 \mathrm{pW}$ or $P R \approx$ $2 \times 10^{8} \mathrm{~s}^{-1}$ at $\lambda=385 \mathrm{~nm}$ ). Similar results were obtained for $d=10 \mathrm{~nm}$ and $\lambda=658 \mathrm{~nm}$.

In figure 6 , the normalized count rate as a function of the wavelength of the incident photons from 600 to $2000 \mathrm{~nm}$ is shown. The spectral count rates have a plateau at lower wavelengths and drop monotonically above the cut-off wavelength.

The data were fitted by

$S D E \propto\left(1+\left(\frac{\lambda}{\lambda_{c}}\right)^{p}\right)^{-1}$

with a cut-off wavelength $\lambda_{\mathrm{c}}$ and an exponent $p$ indicating the decay slope above $\lambda_{\mathrm{c}}$ 31. The highest cut-off wavelength obtained by this fit was about $1248 \mathrm{~nm}$ for $d=5 \mathrm{~nm}\left(I_{\mathrm{b}}=0.90 I_{\text {switch }}\right)$ and $878 \mathrm{~nm}$ for $d=10 \mathrm{~nm}\left(I_{\mathrm{b}}=0.98 I_{\text {switch }}\right)$. 


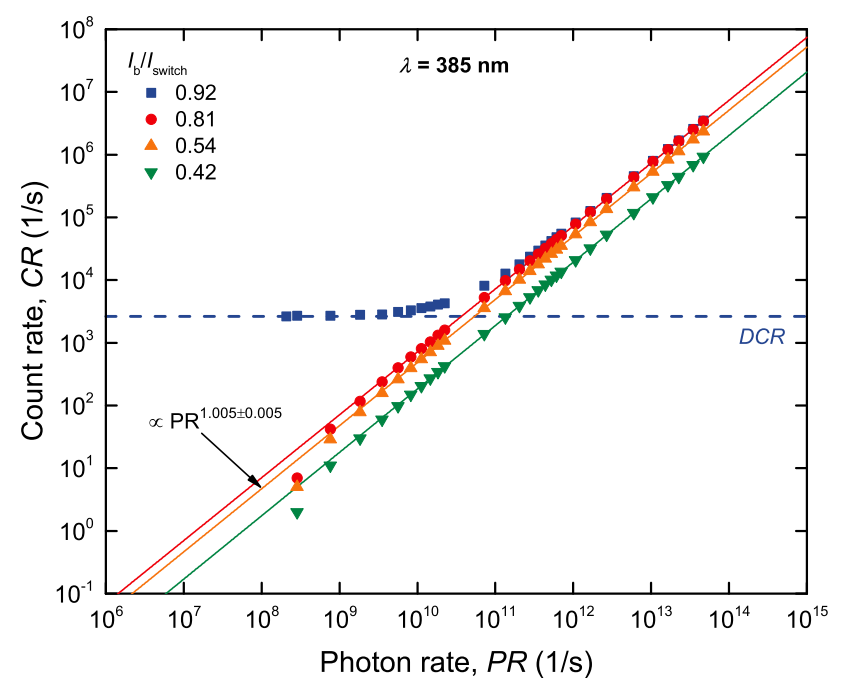

Figure 5: Dependence of the count rate $C R$ on incoming photon rate $P R$ for different bias currents for a detector with thickness $d=5 \mathrm{~nm}$ at $\lambda=385 \mathrm{~nm}$. The solid lines are linear fits to the respective data. The dashed line indicates the level of dark counts at $0.92 I_{\text {switch }}$.

Here, we should note different definitions of the cut-off wavelength. Besides the fit used herein, the intersection of two straight lines is sometimes used, too 31. The latter method, in general, leads to slightly lower values of $\lambda_{c}$.

The estimated timing jitter of the ALD SNSPDs lies well below $28 \mathrm{ps}$, which is the limit of the used experimental setup due to intermodal dispersion in the multimode fiber.

\subsection{Discussion}

According to existing detection models for SNSPDs, events of both photon counts in the probabilistic regime and dark counts could be explained by fluctuations, e.g. vortex hopping [32, 33], vortexantivortex-pair (VAP) unbinding 34 39, or Fano fluctuations 40.

In the framework of these models, the observed low $D C R$ for thicker ALD-NbN films might be explained by the smaller Pearl length, which, in turn, increases the edge barrier for vortex penetration (see equation (20) and (21) in 41]). In addition, the larger volume with the same area of the film-substrate interface of the nanowire should result in smaller thermal fluctuations.

As a second mechanism, dark-counts due to the unbinding of VAPs should be suppressed in $10 \mathrm{~nm}-$ thick nanowires, since the film thickness is more than twice the coherence length $\xi_{\mathrm{GL}}(4.2 \mathrm{~K}) \approx 4.45 \mathrm{~nm}$ and the probability of VAP excitations is therefore lower.

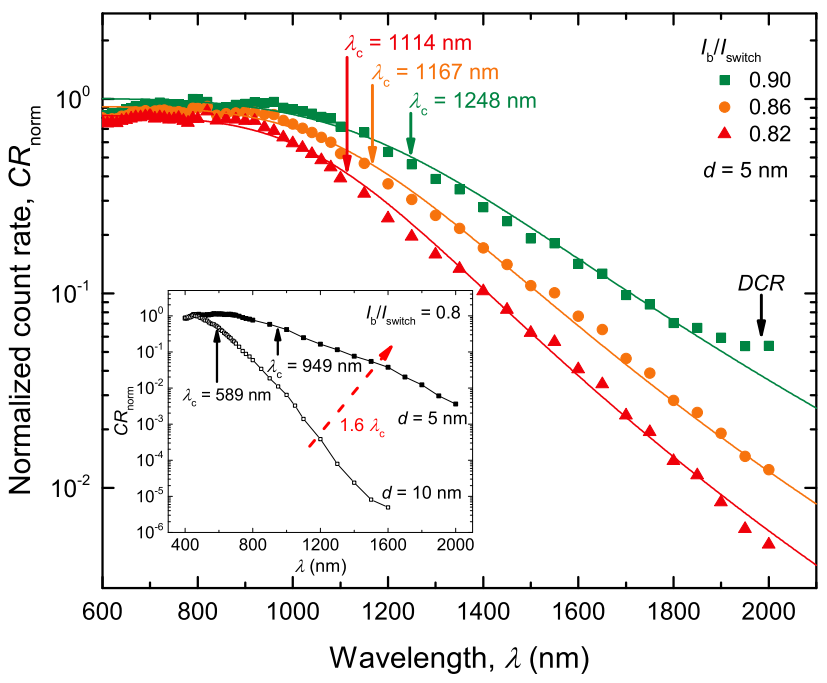

Figure 6: Dependence of the normalized count rate $C R_{\text {norm }}$ on the illumination wavelength $\lambda$ of a $5 \mathrm{~nm}$-thick detector. The solid lines are fits with equation (6). (Inset) Comparison of the normalized count rate $C R_{\text {norm }}$ of 5 and 10 nm-thick detectors with the same $I_{\mathrm{b}} / I_{\text {switch }}$ ratio of 0.8 .

It can be observed, that $\lambda_{c}$ increases with rising bias currents as it is at least qualitatively described by various detection models [8, 29, 36, 42]. According to these models, the superconducting state becomes unstable after the absorption of lower energy photons as the current is increased. This can be explained by the reduced edge-barrier for single vortices and facilitated unbinding of VAPs nucleated inside the hotspot 23, 36, 41. Also, larger portions of the nanowire across the width contribute to the photon detection for increasing bias currents 43 .

Besides that, the detector layout is beneficial for detecting lower-energy photons, since without bends, the single straight nanowires suffer less from currentcrowding and, due to its short length, have a reduced probability of material non-uniformities.

As illustrated by the inset of figure 6 , we get an increase of the cut-off wavelength $\lambda_{\mathrm{c}}$ by a factor of 1.6 for the same ratio $I_{\mathrm{b}} / I_{\text {switch }}$ by reducing the thickness from 10 to $5 \mathrm{~nm}$. This is in line with the expected proportionality $\lambda_{\mathrm{c}} \propto d^{-1}$, since less demand is made for the ratio $I_{\mathrm{b}} / I_{\mathrm{dep}}$ for detectors with smaller crosssections 8,23. A change of $\lambda_{\mathrm{c}}$ by a factor smaller than 2.0 is, at least partly, due to the fact, that material parameters such as $\Delta, D$ and $\tau_{\text {th }}$ also change with the thickness (see equation (4) in [8]). Furthermore, the actual superconducting core of the strip is, relative to its nominal cross-section, smaller for 5 than for $10 \mathrm{~nm}$, as the damaged bands around the superconducting core are expected to remain constant in size. 


\section{Conclusion}

Using the ALD technique, we deposited superconducting $\mathrm{NbN}$ thin films on sapphire substrates. With a small diffusion coefficient $\left(D=0.32 \mathrm{~cm}^{2} \mathrm{~s}^{-1}\right.$ for $d=$ $10 \mathrm{~nm}$ ) and similar values for the ratios $C_{\mathrm{e}} / C_{\mathrm{ph}}$ and $I_{\mathrm{c}} / I_{\mathrm{dep}}(\approx 0.56)$ compared to sputtered $\mathrm{NbN}$, these highly disordered films exhibit well-suited properties for the use in nanowire detectors.

SNSPDs based on this atomic layer-deposited $\mathrm{NbN}$ have been successfully demonstrated. These detectors show saturated $C R(\lambda)$ characteristics and a cut-off wavelength of about $1.25 \mu \mathrm{m}$ for a relatively large cross-section of $5 \mathrm{~nm} \times 100 \mathrm{~nm}$. Decreasing the nanowire width should lead to a further increase of the cut-off wavelength [44.

We expect, that the ALD technology will gain in importance in the future, since it offers greater flexibility regarding substrate materials and wafer size in comparison to existing deposition techniques. In this regard, the homogeneity with respect to both thickness and stoichiometry over large areas should be characterized. The high degree of film uniformity is important for reproducibility and yield of SNSPDs when scaling up to arrays.

\section{Acknowledgments}

The authors acknowledge S. Goerke, K. Pippardt, and U. Hübner for support with the thin film fabrication and S. Linzen for support with the development of the deposition technology.

Part of this work was funded by the Federal Ministry of Education and Research under contract no. $13 \mathrm{~N} 13445$.

\section{Bibliography}

[1] G. N. Gol'tsman, O. Okunev, G. Chulkova, A. Lipatov, A. Semenov, K. Smirnov, B. Voronov, A. Dzardanov, C. Williams, and R. Sobolewski, "Picosecond superconducting single-photon optical detector," Applied Physics Letters, vol. 79, no. 6, pp. 705-707, 2001.

[2] H. Takesue, S. W. Nam, Q. Zhang, R. H. Hadfield, T. Honjo, K. Tamaki, and Y. Yamamoto, "Quantum key distribution over a 40-db channel loss using superconducting single-photon detectors," Nature Photonics, vol. 1, p. 343, 062007.

[3] C. M. Natarajan, M. G. Tanner, and R. H. Hadfield, "Superconducting nanowire single-photon detectors: physics and applications," Superconductor Science and Technology, vol. 25, no. 6, p. 063001, 2012.

[4] J. Toussaint, S. Dochow, I. Latka, A. Lukic, T. May, H.G. Meyer, K. Il'in, M. Siegel, and J. Popp, "Proof of concept of fiber dispersed raman spectroscopy using superconducting nanowire single-photon detectors," Opt. Express, vol. 23, pp. 5078-5090, Feb 2015.

[5] F. Marsili, V. B. Verma, J. A. Stern, S. Harrington, A. E. Lita, T. Gerrits, I. Vayshenker, B. Baek, M. D. Shaw, R. P. Mirin, and S. W. Nam, "Detecting single infrared photons with $93 \%$ system efficiency," Nature Photonics, vol. 7, p. 210, 022013.

[6] B. A. Korzh, Q. Y. Zhao, S. Frasca, J. P. Allmaras, T. M. Autry, E. A. Bersin, M. Colangelo, G. M. Crouch, A. E. Dane, T. Gerrits, et al., "Demonstrating sub-3 ps temporal resolution in a superconducting nanowire single-photon detector," arXiv preprint arXiv:1804.06839, 2018.

[7] A. Migdall, S. V. Polyakov, J. Fan, and J. C. Bienfang, Single-photon generation and detection: physics and applications. Experimental methods in the physical sciences, Amsterdam: Elsevier Academic Press, 2013.

[8] A. Semenov, A. Engel, H.-W. Hübers, K. Il'in, and M. Siegel, "Spectral cut-off in the efficiency of the resistive state formation caused by absorption of a single-photon in current-carrying superconducting nanostrips," The European Physical Journal B - Condensed Matter and Complex Systems, vol. 47, pp. 495-501, Oct 2005.

[9] S. N. Dorenbos, P. Forn-Díaz, T. Fuse, A. H. Verbruggen, T. Zijlstra, T. M. Klapwijk, and V. Zwiller, "Low gap superconducting single photon detectors for infrared sensitivity," Applied Physics Letters, vol. 98, no. 25, p. 251102, 2011.

[10] A. J. Kerman, E. A. Dauler, J. K. W. Yang, K. M. Rosfjord, V. Anant, K. K. Berggren, G. N. Gol'tsman, and B. M. Voronov, "Constriction-limited detection efficiency of superconducting nanowire single-photon detectors," Applied Physics Letters, vol. 90, no. 10, p. 101110, 2007.

[11] I. Charaev, T. Silbernagel, B. Bachowsky, A. Kuzmin, S. Doerner, K. Ilin, A. Semenov, D. Roditchev, D. Y. Vodolazov, and M. Siegel, "Enhancement of superconductivity in nbn nanowires by negative electronbeam lithography with positive resist," Journal of Applied Physics, vol. 122, no. 8, p. 083901, 2017.

[12] B. Baek, A. E. Lita, V. Verma, and S. W. Nam, "Superconducting a-wxsi1-x nanowire single-photon detector with saturated internal quantum efficiency from visible to $1850 \mathrm{~nm}$," Applied Physics Letters, vol. 98, no. 25, p. $251105,2011$.

[13] V. B. Verma, B. Korzh, F. Bussières, R. D. Horansky, S. D. Dyer, A. E. Lita, I. Vayshenker, F. Marsili, M. D. Shaw, H. Zbinden, R. P. Mirin, and S. W. Nam, "High-efficiency superconducting nanowire single-photon detectors fabricated from mosi thin-films," Opt. Express, vol. 23, pp. 33792-33801, Dec 2015.

[14] D. Morozov, A. Banerjee, K. Erotokritou, G. Taylor, N. R. Gemmell, D. Hemakumara, I. Thayne, and R. H. Hadfield, "Superconducting nanowire materials for mid infrared single photon detection (conference presentation)," Proc. SPIE, vol. 10659, 2018.

[15] M. Ziegler, L. Fritzsch, J. Day, S. Linzen, S. Anders, J. Toussaint, and H.-G. Meyer, "Superconducting niobium nitride thin films deposited by metal organic plasmaenhanced atomic layer deposition," Superconductor Science and Technology, vol. 26, no. 2, p. 025008, 2012.

[16] M. Ziegler, S. Linzen, S. Goerke, U. Brückner, J. Plentz, J. Dellith, A. Himmerlich, M. Himmerlich, U. Hübner, S. Krischok, and H. G. Meyer, "Effects of plasma parameter on morphological and electrical properties of superconducting nb-n deposited by mo-peald," IEEE Transactions on Applied Superconductivity, vol. 27, pp. 1-7, Oct 2017.

[17] S. Linzen, M. Ziegler, O. V. Astafiev, M. Schmelz, U. Hübner, M. Diegel, E. Il'ichev, and H.-G. Meyer, "Structural and electrical properties of ultrathin niobium nitride films grown by atomic layer deposition," Superconductor Science and Technology, vol. 30, no. 3, p. 035010, 2017.

[18] D. Henrich, S. Dörner, M. Hofherr, K. Il'in, A. Semenov, 
E. Heintze, M. Scheffler, M. Dressel, and M. Siegel, "Broadening of hot-spot response spectrum of superconducting nbn nanowire single-photon detector with reduced nitrogen content," Journal of Applied Physics, vol. 112 , no. 7, p. $074511,2012$.

[19] A. Semenov, B. Günther, U. Böttger, H.-W. Hübers, H. Bartolf, A. Engel, A. Schilling, K. Ilin, M. Siegel, R. Schneider, D. Gerthsen, and N. A. Gippius, "Optical and transport properties of ultrathin nbn films and nanostructures," Phys. Rev. B, vol. 80, p. 054510, Aug 2009.

[20] U. S. Pracht, E. Heintze, C. Clauss, D. Hafner, R. Bek, D. Werner, S. Gelhorn, M. Scheffler, M. Dressel, D. Sherman, B. Gorshunov, K. S. Il'in, D. Henrich, and M. Siegel, "Electrodynamics of the superconducting state in ultra-thin films at thz frequencies," IEEE Transactions on Terahertz Science and Technology, vol. 3, pp. 269-280, May 2013.

[21] H. Bartolf, Fluctuation Mechanisms in Superconductors. Wiesbaden: Springer Spektrum, 1st ed. 2016 ed., 2016.

[22] N. R. Werthamer, E. Helfand, and P. C. Hohenberg, "Temperature and purity dependence of the superconducting critical field, $H_{c 2}$. iii. electron spin and spin-orbit effects," Phys. Rev., vol. 147, pp. 295-302, Jul 1966.

[23] D. Y. Vodolazov, "Single-photon detection by a dirty current-carrying superconducting strip based on the kinetic-equation approach," Phys. Rev. Applied, vol. 7, p. 034014, Mar 2017.

[24] J. Pearl, "Current distribution in superconducting films carrying quantized fluxoids," Applied Physics Letters, vol. 5, no. 4, pp. 65-66, 1964.

[25] K. Il'in, M. Siegel, A. Semenov, A. Engel, and H.W. Hübers, "Critical current of nb and nbn thinfilm structures: The cross-section dependence," physica status solidi (c), vol. 2 , no. 5, pp. 1680-1687, 2005.

[26] M. Kupriyanov and V. Lukichev, "Temperature dependence of pair-breaking current in superconductors," Sov. J. Low Temp. Phys. (Engl. Transl.), vol. 6, 41980.

[27] Y. V. Fominov and M. V. Feigel'man, "Superconductive properties of thin dirty superconductor-normal-metal bilayers," Phys. Rev. B, vol. 63, p. 094518, Feb 2001.

[28] H. L. Hortensius, E. F. C. Driessen, T. M. Klapwijk, K. K. Berggren, and J. R. Clem, "Critical-current reduction in thin superconducting wires due to current crowding," Applied Physics Letters, vol. 100, no. 18, p. 182602, 2012.

[29] D. Y. Vodolazov, "Current dependence of the red boundary of superconducting single-photon detectors in the modified hot-spot model," Phys. Rev. B, vol. 90, p. 054515, Aug 2014.

[30] Y. Korneeva, M. Sidorova, A. Semenov, S. Krasnosvobodtsev, K. Mitsen, A. Korneev, G. Chulkova, and G. Goltsman, "Comparison of hot-spot formation in nbc and nbn single-photon detectors," IEEE Transactions on Applied Superconductivity, vol. 26, pp. 1-4, April 2016.

[31] I. Charaev, A. Semenov, S. Doerner, G. Gomard, K. Ilin, and M. Siegel, "Current dependence of the hot-spot response spectrum of superconducting single-photon detectors with different layouts," Superconductor Science and Technology, vol. 30, no. 2, p. 025016, 2017.

[32] L. N. Bulaevskii, M. J. Graf, and V. G. Kogan, "Vortex-assisted photon counts and their magnetic field dependence in single-photon superconducting detectors," Phys. Rev. B, vol. 85, p. 014505, Jan 2012.

[33] A. N. Zotova and D. Y. Vodolazov, "Intrinsic detection efficiency of superconducting nanowire single photon detector in the modified hot spot model," Superconductor Science and Technology, vol. 27, no. 12, p. 125001, 2014.

[34] A. N. Zotova and D. Y. Vodolazov, "Photon detection by current-carrying superconducting film: A timedependent ginzburg-landau approach," Phys. Rev. B, vol. 85, p. 024509, Jan 2012.

[35] A. Engel, J. J. Renema, K. Il'in, and A. Semenov, "Detection mechanism of superconducting nanowire single-photon detectors," Superconductor Science and Technology, vol. 28, no. 11, p. 114003, 2015.

[36] D. Y. Vodolazov, Y. P. Korneeva, A. V. Semenov, A. A. Korneev, and G. N. Goltsman, "Vortex-assisted mechanism of photon counting in a superconducting nanowire single-photon detector revealed by external magnetic field," Phys. Rev. B, vol. 92, p. 104503, Sep 2015.

[37] A. Engel, A. Semenov, H.-W. Hübers, K. Ilin, and M. Siegel, "Fluctuations and dark count rates in superconducting nbn single-photon detectors," physica status solidi (c), vol. 2, no. 5, pp. 1668-1673, 2005.

[38] J. Kitaygorsky, I. Komissarov, A. Jukna, D. Pan, O. Minaeva, N. Kaurova, A. Divochiy, A. Korneev, M. Tarkhov, B. Voronov, I. Milostnaya, G. Gol'tsman, and R. R. Sobolewski, "Dark counts in nanostructured nbn superconducting single-photon detectors and bridges," IEEE Transactions on Applied Superconductivity, vol. 17, pp. 275-278, June 2007.

[39] T. Yamashita, S. Miki, K. Makise, W. Qiu, H. Terai, M. Fujiwara, M. Sasaki, and Z. Wang, "Origin of intrinsic dark count in superconducting nanowire single-photon detectors," Applied Physics Letters, vol. 99, no. 16, p. $161105,2011$.

[40] A. G. Kozorezov, C. Lambert, F. Marsili, M. J. Stevens, V. B. Verma, J. P. Allmaras, M. D. Shaw, R. P. Mirin, and S. W. Nam, "Fano fluctuations in superconductingnanowire single-photon detectors," Phys. Rev. B, vol. 96, p. 054507, Aug 2017.

[41] H. Bartolf, A. Engel, A. Schilling, K. Il'in, M. Siegel, H.-W. Hübers, and A. Semenov, "Current-assisted thermally activated flux liberation in ultrathin nanopatterned nbn superconducting meander structures," Phys. Rev. B, vol. 81, p. 024502, Jan 2010.

[42] L. Maingault, M. Tarkhov, I. Florya, A. Semenov, R. Espiau de Lamaëstre, P. Cavalier, G. Gol'tsman, J.-P. Poizat, and J.-C. Villégier, "Spectral dependency of superconducting single photon detectors," Journal of Applied Physics, vol. 107, no. 11, p. 116103, 2010.

[43] J. J. Renema, Q. Wang, R. Gaudio, I. Komen, K. op 't Hoog, D. Sahin, A. Schilling, M. P. van Exter, A. Fiore, A. Engel, and M. J. A. de Dood, "Position-dependent local detection efficiency in a nanowire superconducting single-photon detector," Nano Letters, vol. 15, no. 7, pp. 4541-4545, 2015.

[44] F. Marsili, F. Bellei, F. Najafi, A. E. Dane, E. A. Dauler, R. J. Molnar, and K. K. Berggren, "Efficient single photon detection from $500 \mathrm{~nm}$ to $5 \mu \mathrm{m}$ wavelength," Nano Letters, vol. 12, no. 9, pp. 4799-4804, 2012. 